\title{
802.11 Link Quality and Its Prediction - An Experimental Study
}

\author{
Gregor Gaertner, Eamonn ONuallain, Andrew Butterly, Kulpreet Singh, and \\ Vinny Cahill \\ Distributed Systems Group \\ Department of Computer Science \\ Trinity College, Dublin, Ireland \\ gregor.gaertner@cs.tcd.ie
}

\begin{abstract}
Reliable link quality prediction is an imperative for the efficient operation of mobile ad-hoc wireless networks (MANETs). In this paper it is shown that popular link quality prediction algorithms for 802.11 MANETs perform much more poorly when applied in real urban environments than they do in corresponding simulations. Our measurements show that the best performing prediction algorithm failed to predict between 18 and 54 percent of the total observed packet loss in the real urban environments examined. Moreover, with this algorithm between 12 and 43 percent of transmitted packets were lost due to the erroneous prediction of link failure. This contrasts sharply with near-perfect accuracy in corresponding simulations. To account for this discrepancy we perform an in-depth examination of the factors that influence link quality. We conclude that shadowing is an especially significant and hitherto underestimated factor in link quality prediction in MANETs.
\end{abstract}

\section{Introduction}

With the deployment of MANETs having just begun, practical experience of wireless protocol performance is limited. A-priori performance analysis of MANET protocols is difficult due to multi-hop communication with intractable and environment-specific signal propagation effects. Hence provably reliable communication is still in its infancy [1] and network performance evaluations are largely based on simulations. Recent studies however have cast serious doubts on the reliability of results obtained from such simulations. Discrepancies between simulated and real-world network performance has been primarily attributed due to the inappropriate level of detail at which these simulations are performed including the use of overly-simplified propagation models 234 .

The propagation models commonly used in such simulations are based on very 'benign' obstacle-free environments. Obstructing static and dynamic objects (e.g. buildings, people, cars, etc.) as well as important radio propagation effects (diffraction, scattering and transmission 5]) are not considered. These are major shortcomings since the primary deployment areas for MANETs are urban environments where such effects are pronounced. Since the network topology 
and environment change rapidly in MANETs, the communication quality varies considerably with time. Link quality prediction is a key technique to alleviate link quality degradations by pro-actively adapting the network to the operating environment.

We now evaluate the performance of some of the more popular link quality prediction algorithms for MANETs in two real urban environments. Our results indicate that these algorithms perform poorly in such a setting despite having exhibited near-perfect performance in simulations. While some performance degradation is to be expected when such algorithms are applied in real environments, the observed discrepancy is much larger than expected. This motivates us to systematically identify the factors that influence link quality (henceforth called 'influencing factors') and assess their importance for link quality prediction so that these discrepancies can be accounted for.

The remainder of this paper is organized as follows: Section 2 discusses current work on link quality prediction and its applications. Section 3 evaluates the accuracy of current link quality prediction algorithms in a real-world case study and compares their performance with simulations. Improvements for future algorithms are recommended. Section 4 identifies the factors influencing link quality and assesses their importance in prediction accuracy. We conclude our work in Sect. 5.

\section{Related Work}

It has been shown in numerous simulations that link quality prediction is important for routing in ad-hoc networks. In wired networks the shortest distance criterion is often used to select optimal paths for routing. However, as a result of the significantly higher number of link failures in wireless ad-hoc networks, a more sophisticated means is required for route optimisation in these networks. Using link quality prediction methods, the protocols of 6 6789,101112 can select routes with higher lifetimes. To reduce traffic on shared low bandwidth channels on-demand routing protocols, which only initiate route discovery for active paths, are used. If a path breaks, high latency is the costly outcome due to multiple timeouts. Proactive route discovery enabled by link quality prediction is utilized in 8912 13 14 to alleviate this problem by initiating route discovery before the path breaks. This approach reduces packet loss and jitter. Link quality prediction may also be used to dynamically cluster the network into groups of stable nodes. This reduces the update propagation time of changes in network topology 15. Another application of pro-active failure detection is enabling consistency maintenance in group communication $[16$.

Link quality prediction algorithms can be categorized as follows: deterministic approaches 6.78910 12 1314 which give a precise value for link quality and stochastic approaches 11 17 18 which give a probabilistic measure. All approaches (with the exception of [17]) employ a deterministic signal propagation model either explicitly or implicitly. The signal propagation models in common usage are the simple Radial Model of [16,18; the assumption that the trans- 
mitted power and the distance separating the nodes alone determines the signal strength [6.710]; the Free Space Propagation Model [19, pg. 107-109] used in 912] and the Two-Ray Ground Model [19. pg. 120-125] used in 81314. At runtime 6 7 10,13:14] use signal strength criteria to provide an estimate for link quality whereas 89:12,20] use node location for this purpose. All approaches are evaluated by simulation with either NS-2 [21] or GloMoSim [22] except for [8] which is evaluated experimentally.

It should be noted that in the above link quality prediction algorithms the link quality metric is determined primarily from the distance between nodes. Though this may hold for very benign open-space environments, it breaks down in real urban environments where there are substantial signal fading effects due to manifold signal propagation phenomena. In the above works only simple techniques, if any, have been suggested for dealing with fast signal fading (e.g. exponential average [6], linear regression [14] or the ping-pong mechanism of [13]). Consequently these approaches exhibit a significantly poorer performance when applied in real-world urban scenarios over simulations (see Sect. 3).

In [6] the signal strength is measured and the link is classified simply as being either strong or weak. An 'affinity metric' proposed in [7] and later used in [10] gives in contrast a continuous measure for the link quality which is determined primarily from the trend of the most recent samples of the signal strength.

In 13 a pre-emptive threshold is compared with the current signal strength. If the signal strength is lower than this threshold, the possibility of link failure is considered. This leads to an exchange of a pre-set number of messages called ping-pong rounds. If the signal strength of greater than a certain number of these packets is under the threshold, then a link failure is predicted. This mechanism aims to reduce the number of link failures which are predicted erroneously due to fast signal fading. The pre-emptive threshold is calculated using the node transmission range and the Two-Ray Ground Model so that there is enough time to establish a potential alternative route before the link fails. Regrettably an appropriate threshold is difficult to calculate in a real-world environment since the range of the nodes is environment-specific and unknown.

In 14 the Law of Cosines is used to derive the remaining time to link failure. This prediction algorithm is based on the last three received signal strength samples, the Two-Ray Ground Model and assumptions of the Random Waypoint Mobility Model [23. Linear regression is suggested to pre-process the signal strength values in order to counter the effects of fast signal fading. The algorithm assumes that nodes have a constant velocity and that the signal strength is affected only by the distance between nodes.

Node location and velocity are used with the Free-Space Propagation Model in 912 to predict the time to link failure. This propagation model is inappropriate even for open-space environments (see [19, pg. 120]) and the need for location and velocity information makes the algorithm costly to implement. The prediction method suggested in [8] consists of modules for mobility prediction, signal strength prediction and an environment map. The future signal strength is determined from the predicted distance between the nodes, an 
experimentally-determined site-specific coefficient for shadowing and the TwoRay Ground Model. The concept is evaluated experimentally in a parking lot. Unfortunately the graphs given in the paper use a time scale that precludes a direct evaluation of the prediction accuracy in the order of seconds. The algorithm is critically dependent on the distance between nodes determined via the absolute node positions without tolerating missing position data. This is a shortcoming since the suggest positioning system GPS is often unreliable in dense urban environments (this is confirmed by our measurements in Sect. [3.1). Furthermore it is not clear whether memory consuming maps of the environment with their associated computationally intensive algorithms justify their cost since unaccounted for moving objects have a strong bearing on link quality (see Sect. 4.2).

Three stochastic approaches have been proposed. [1118] predict link failures based on the Radial Propagation Model and the mobility pattern of the Random Ad-hoc Mobility Model [18]. It is unclear how well these approaches work in practice, especially since very simple propagation models are employed. In [17] links with an expected higher remaining lifetime are chosen based on previous link lifetimes. It is unclear weather this concept can be used for link failure prediction.

The handoff problem (see the survey article [24] for example) in cellular communication networks is analogous to the link quality prediction problem in MANETs. Based on the current mobility pattern it is attempted to execute optimal handoff from one cell to another such that potential consequent disruptions are minimized. While this problem has been well studied, the suggested solutions are only partially transferable to link quality prediction in MANETs. For a detailed discussion see Sect. 4.2.

Since the literature on link quality prediction focuses on simulations as the means for the evaluation of algorithms, it is clear that the accuracy of the used simulators is crucial. 2] compares popular simulators by their physical layer models, their implementations and a case study. This study revealed that the evaluation of protocols in different simulators may give different absolute and even different relative performance measures. In [3] similar results were reported for a simple broadcast protocol implemented by flooding. Since broadcast protocols are used as basic building blocks for many wireless protocols, [3] concludes that "finally, beside simulations and according to the feeling of the MANET community, there is an important lack of real experiments that prove the feasibility of wireless protocols".

\section{Accuracy of Link Quality Prediction in the Real-World}

In this section we evaluate the accuracy of some popular link quality prediction algorithms experimentally in two typical urban environments. We then compare the results obtained with those derived by simulations. 


\subsection{Case Study and Test Bed Description}

The urban environments we chose include a variety of static (e.g. buildings, trees, etc.) and dynamic objects (people, cars). The selected locations in Dublin's city centre are fairly typical of European cities. Grafton Street is a long narrow pedestrian precinct overshadowed by moderately high (three-storey) buildings. There is no vegetation and it is commonly quite full of people. The second location, O'Connell Street, is a wide avenue-like street. There are two lanes of traffic in both directions. These are separated by a verge with some trees. Traffic is heavy throughout the day. On both sides of the street are sidewalks which are normally full of pedestrians. We conducted our experiments at busy times. We will refer to Grafton Street as 'Street 1' and O'Connell Street as 'Street 2' in this paper.

Shadowing and propagation effects are the prominent factors bearing on link quality in urban environments over the occurrence of collisions in the presence of a moderate number of nodes. Hence, working with two nodes, we consider the former effects only. In our study the bearers of both nodes moved between randomly chosen shops, their movement interspersed with pauses of about two seconds as might be expected of some pedestrians on these streets. This mobility pattern corresponds to the widely used Random Waypoint Mobility Model with a maximum speed of $1.5 \mathrm{~m} / \mathrm{s}$ (walking speed) using pauses of two seconds. The movement area of the nodes was confined to the main street and its immediate precinct to ensure a certain level of connectivity.

Both nodes were broadcasting messages of 100 bytes at regular intervals (heartbeats) directly over the MAC layer without using any higher-level protocol at a transmission rate of $2 \mathrm{Mbit}$. The coherence time of $17.63 \mathrm{~ms}$ for the speed of our nodes suggests a sampling rate of greater than $28.36 \mathrm{~Hz}$ if multi-path effects are to be fully captured by the measurements. However, the experience of the cellular network community shows that the incorporation of multi-path fading is impractical for prediction algorithms (see [25] for example) and the prediction algorithms evaluated in this work do not incorporate such information. Consequently, in order to reduce the amount of data, we use a sample rate of $10 \mathrm{~Hz}$ which allows for prediction in convenient $100 \mathrm{~ms}$ intervals. We conducted three trials of 30 minutes duration for each street. All sent and received packets were recorded together with their associated average signal strength.

Two Dell Latitude C400 notebooks with Lucent Orinoco Gold wireless $802.11 \mathrm{~b}$ cards acted as nodes. The transmission power was set to the maximum of $15 \mathrm{dBm}$ with disabled power management. We used Redhat Linux 7.3 together with the GPL orinoco_cs driver by David Gibson in version 0.12 [26], which we extended to send and receive packets directly over the MAC layer. During the trials it was attempted to record the current node positions with either a Magellan GPS 315 or a Garmin ETrax receiver. However, the number of satellites in view was mostly insufficient to obtain a position.

For the simulations NS-2 was configured according to the real world study. The movements of the two nodes followed the Random Waypoint Mobility Model with a maximum speed $1.5 \mathrm{~m} / \mathrm{s}, 2$ seconds pause and an area of $500 \mathrm{~m}$ squared. 
The Two-Ray Ground Model and the parameters of a Lucent Wavelan card with a maximum range of $250 \mathrm{~m}$ were employed. Our choice of mobility and propagation models corresponds to most MANET simulations (see 2101314] for examples).

\subsection{Algorithm Implementations}

We restrict our evaluation to algorithms with the following properties:

1. The algorithm predicts at time $t$ if the link is either available or unavailable at time $t+T_{\mathrm{p}}$ ( $T_{\mathrm{p}}$ is called the prediction horizon). Most pro-active applications in routing or group communication (see [71314]16] for examples) require this very weak property ('weak' since only a binary value for the link quality at only one point in time is used).

2. The algorithms must tolerate missing node locations for long time periods since GPS signal reception can be very unreliable in dense urban environments.

3. The signal propagation model used must be at least as accurate as the TwoRay Ground Model. Even this model does not account for the manifold propagation phenomena in urban environments.

These criteria that aim to identify the most likely successful algorithms in urban environments reduce our evaluation to [713:14.

In order to unify the notation for the algorithms considered, we use the following symbols: a lower case ' $t$ ' denotes a point in time, ' $T$ ' denotes a time interval. ' $P_{\mathrm{t}}$ ' denotes the signal strength of a packet received at time $t$. ' $P$ maxrange' denotes the minimum signal strength at which a packet at the maximum distance maxrange can be received. Threshold values used in the algorithms are denoted using the symbol ' $\delta$ '. The symbol ' $\Delta$ ' is used to denote the difference between successive values of a variable.

The Affinity Algorithm. The Affinity Algorithm originating in [7] and applied in [10], associates a link with an affinity metric $a$. The algorithm assumes that the mobility pattern remains constant and that the signal strength increases if and only if the nodes move closer and decreases if the nodes move further apart. Thus, if the average signal trend $\Delta P_{[t-n+1, t]}$ over $n$ samples remains positive, the nodes are assumed to move closer and the affinity is classified as being 'high'. The link is predicted to remain available in this case. However, if the signal trend is negative, the nodes are assumed to be moving apart and the affinity at time $t$ for a horizon $T_{\mathrm{p}}$ is calculated via $a_{t}=\frac{P_{\operatorname{maxrange}}-P_{\mathrm{t}}}{\Delta P_{[t-n+1, t]}}$. Under the condition that all assumptions are true the affinity value is a direct measure of the time to link failure.

Our implantation predicts a link failure at $t$ for $t+T_{\mathrm{p}}$ if the affinity value is below a threshold $a_{\delta}$. For best performance in the real world we determine the parameter values $a_{\delta}$ and $n$ using an optimization routine (see Sect. 3.3 for details). An analytical determination of these parameters based on the original work gives good performance results in simulations only. 
The Law of Cosines (LoC) Algorithm. This algorithms 14] calculates the time to link failure based on the signal strength values of three consecutive received packets. The algorithm assumes that the signal strength follows strictly the Two-Ray Ground Model and that nodes maintain a constant velocity until the predicted link failure occurs. The algorithm is based on a simple geometric evaluation using Law of Cosines. A link is predicted to be available if the signal strength over three consecutive received packets is either constant or increasing. Otherwise a time to link failure is predicted.

Fast signal fading effects are attempted to be masked using linear regression. However, the signal strength may still differ greatly with that given by the Two Ray Ground model even when using an optimum window-size and so the quadratic equation used to give link failure time occasionally gives an imaginary solution. This problem was not observed during simulation.

The Pre-emptive Threshold Algorithm. The above algorithms use the recent signal trend as their link quality metric. In contrast, [13] uses a combination of the signal strength itself and the recent packet loss. If the current signal strength is under $P_{\delta}$, a link failure is suspected. As result packets are exchanged in $n$ ping-pong rounds between the two nodes. If $k \leq n$ packets are below the signal strength threshold $P_{\delta}$, a link failure is predicted. This method attempts to overcome erroneous prediction of link failures due to fast signal fading by tolerating adverse link quality fluctuations over $k-1$ packets. The threshold of the signal strength $P_{\delta}$ is determined from the so called pre-emptive threshold as $\delta=\left(\frac{\text { maxnoderange }}{\text { maxnoderange-maxrelspeed } T_{\mathrm{p}}}\right)^{4}$. The threshold attempts to enable a communication task to be completed even if two nodes move away with the maximum speed maxrelspeed. However, this is only achieved if the signal strength behaves strictly according to the Two-Ray Ground Model and the maximum transmission range maxrange is known.

If in our implementation the signal strength value associated with one packet is below $P_{\delta}$, a node counts how many out of the next $n$ received heartbeat packets have also a signal strength value below $P_{\delta}$. If this number is equal or greater than $k$, a link failure is predicted. We calculated the threshold values for the simulation as outlined in the original work and set $n=k=3$. However, for the real-world study these parameters are unknown, especially the environment specific average maximum transmission range. Consequently we determined all parameter values by optimization as described in the next section.

\subsection{Evaluation Metrics and Results}

In current simulators link failures are modelled as being 'sharp'. This contrasts with the real world behaviour, where a link typically oscillates between being available and unavailable over a transitional period before eventually remaining unavailable. Hence a definition of link stability is necessary. We define a link to be stable in a system with a constant heartbeat rate, if at least $p$ percent of packets are received during a time span $T_{\mathrm{s}} . T_{\mathrm{s}}$ should be small enough to reflect 
fast changes of the link status but large enough to tolerate fast-fading effects (i.e., to prevent unnecessary route discoveries in routing protocols). $p$ must be small enough to meet the minimum link quality requirements of an application and large enough to tolerate fast signal fading. Our results suggest $p=70 \%$ and $T_{\mathrm{s}}=2 \mathrm{~s}$ are reasonable values. Since all algorithms considered here are based on the implicit assumption that a link fails sharply, we only measure the prediction accuracy outside the transitional period.

We define our accuracy metrics for link quality prediction in like manner with those used in handoff algorithms in cellular networks (i.e., service failure and unnecessary handoffs 25): the percentage of packets that are lost while the link was predicted to be available (henceforth called 'missed packet loss') and the percentage of packets that are received while the link was predicted to be unavailable (henceforth called 'unnecessary packet loss'). These metrics capture the fundamental trade-off in link failure prediction: if the prediction is too pessimistic, the unnecessary packet loss is high but the missed packet loss is low. If the prediction is too optimistic the reverse is the case. Since both metrics have an equal bearing on performance, an overall accuracy metric must include both. We define the degree of prediction inaccuracy $i=m^{2}+u^{2}+(m-u)^{2}$ as the sum of the squares of missed packet loss $m$ and unnecessary packet loss $u$ with an additional term that accounts for an imbalance of these two metrics.

As stated earlier, suitable parameter values for the prediction algorithms must be determined for the environment in question. Clearly the way to do this is to minimise the inaccuracy $i$. This is, however, a difficult task since the inaccuracy function associated with a prediction algorithm and dataset is not analytically differentiable and also highly non-linear. The parameter space is cleft so that even classical direct search algorithms perform inadequately since they get stuck at local minima as a result of the greedy criterion. We resolved these problems using a robust evolutionary optimization algorithm - The Differential Evolution Algorithm [27.

Table 1 displays the performance of all the algorithms considered under different criteria. 'MPL' stands for the missed packet loss, 'UPL' - the unnecessary packet loss and 'OI' - the overall inaccuracy. The table is divided into separate sections corresponding to real-world experiments and simulation. The experimental section is further partitioned into data sets on which the optimization of the algorithm parameters was conducted. This distinction is required since the optimum parameters are environment-specific. Optimization (Opt.) 1 and 2 refers to minimizing the inaccuracy based on either the data set for Street 1 or 2 respectively. Opt. 3 refers to minimization of the inaccuracy on a compound data set of Street 1 and 2 that aims to find 'compromise' parameter values for both environments. In the simulation study optimization is not required since the parameters are determined analytically.

The table shows that in our real-world measurements the Pre-Emptive Threshold Algorithm achieves the best accuracy by far. However 22.85/28.39\% of missed packet loss and $28.07 / 30.50 \%$ of unnecessary packet loss was measured in Street $1 / 2$ despite optimal parameters having been used for the environment in 
Table 1. Prediction accuracy in real-world urban environments and in simulations

\begin{tabular}{|c|c|c|c|c|c|c|c|c|}
\hline & \multicolumn{3}{|c|}{ Affinity Algorithm } & \multicolumn{2}{|l|}{ LoC Algorithm } & \multicolumn{3}{|c|}{$\begin{array}{l}\text { Pre-Emptive Threshold } \\
\text { Algorithm }\end{array}$} \\
\hline \multicolumn{9}{|c|}{ Experimental Trial - Street 1 (Grafton Street) } \\
\hline & Opt. 1 & Opt. 2 & Opt. 3 & \begin{tabular}{|l|l|} 
Opt. 1 & Opt. 2 \\
\end{tabular} & Opt. 3 & Opt. 1 & Opt. 2 & Opt. 3 \\
\hline MPL & $48.72 \%$ & $49.63 \%$ & $49.45 \%$ & $58.17 \%$ & $58.17 \%$ & $22.85 \%$ & $53.71 \%$ & $31.73 \%$ \\
\hline UPL & $34.73 \%$ & $34.77 \%$ & $34.77 \%$ & \begin{tabular}{l|l}
$27.35 \%$ & $22.71 \%$
\end{tabular} & $27.35 \%$ & $28.07 \%$ & $11.72 \%$ & $22.62 \%$ \\
\hline OI & 3775.53 & 3892.91 & 3869.76 & \begin{tabular}{|l|l|}
5081.64 & 6418.15 \\
\end{tabular} & 5081.64 & 1337.30 & 4785.28 & 1601.45 \\
\hline \multicolumn{9}{|c|}{ Experimental Trial - Street 2 (O'Connell Street) } \\
\hline & Opt. 1 & Opt. 2 & Opt. 3 & \begin{tabular}{|l|l|} 
Opt. 1 & Opt. 2 \\
\end{tabular} & Opt. 3 & Opt. 1 & Opt. 2 & Opt. 3 \\
\hline MPL & $55.59 \%$ & $54.19 \%$ & $54.19 \%$ & $61.75 \%$ & $61.75 \%$ & $17.81 \%$ & $28.39 \%$ & $19.43 \%$ \\
\hline $\mathrm{UPL}$ & $36.89 \%$ & $35.90 \%$ & $35.91 \%$ & $27.40 \%$ & $27.40 \%$ & $43.40 \%$ & $30.50 \%$ & $39.06 \%$ \\
\hline $\mathrm{OI}$ & 4800.81 & 4559.89 & 4560.24 & \begin{tabular}{|l|l|}
5743.75 & 5571.13 \\
\end{tabular} & 5743.75 & 2855.60 & 1740.69 & 2288.55 \\
\hline \multicolumn{9}{|c|}{ Simulation } \\
\hline & \multicolumn{3}{|c|}{ Calculated } & \multicolumn{2}{|c|}{ Calculated } & \multicolumn{3}{|c|}{ Calculated } \\
\hline MPL & \multicolumn{3}{|c|}{$0.00 \%$} & \multicolumn{2}{|c|}{$6.67 \%$} & \multicolumn{3}{|c|}{$0.00 \%$} \\
\hline $\mathrm{UPL}$ & \multicolumn{3}{|c|}{$0.00 \%$} & \multicolumn{2}{|l|}{$0.00 \%$} & \multicolumn{3}{|c|}{$0.00 \%$} \\
\hline OI & \multicolumn{3}{|c|}{0.00} & \multicolumn{2}{|l|}{44.44} & \multicolumn{3}{|c|}{0.00} \\
\hline
\end{tabular}

question. This performance decreases with the use of non-optimum parameters that have been obtained in similar environments. For example, the accuracy of the Pre-Emptive Threshold Algorithm deteriorated in Street 1 from 1337.30 to 4785.28 when the algorithm parameters used were the optimum parameters for Street 2. These results indicate that an online learning is necessary that adapts the parameters to the current operating environment.

The Affinity and LoC Algorithms performed much more poorly on all counts than the Pre-emptive Threshold algorithm. Both algorithms failed to predict approximately half the packet loss while a quarter to one third of packets was unnecessarily predicted to be lost. We attribute this poor accuracy to these algorithms' reliance on signal trend data which is highly sensitive to fast-fading effects. The mechanism of linear regression employed in the LoC Algorithm and the window-size used in the Affinity Algorithm seem both to be ineffective in dealing with fast signal fading. The Pre-Emptive Threshold algorithm shows a greater robustness in this regard since it uses the current signal strength values and not signal trend. This observation demonstrates that signal strength is a more powerful predictor of packet loss if the path loss in the prediction interval is insignificant like it is the case for low node speeds.

In the simulation all algorithms show near-perfect accuracy, both in terms of missed and unnecessary packet loss. Only the LoC Algorithm fails to predict some lost packets. This occurs in situations where the node speed changed during the sampling interval of the three consecutive packets used for prediction (as described in [14). We would have liked to have compared the results of our simulations directly with those of the original authors. However [7,13 assessed the prediction accuracy indirectly using metrics that show the enhancement of the routing protocol performance due to link failure prediction. [14, nevertheless, 
gives explicit prediction accuracies where it is reported that more than 90 percent of lost packets were predicted successfully for various mobility patterns. We observed a value of 93 percent in our simulation.

The near-perfect accuracy in simulations can be attributed to the fact that the prediction algorithms and simulation programs use the same deterministic radio propagation model. It is well known that the propagation models used by such simulators are simple and yield only to real-world approximations (see [5] for example). Thus simulation accuracy is perceived as being optimistic (see [14] for example). However, the large discrepancy between link quality prediction accuracy given by simulators and those obtained in real-world trials has apparently not been appreciated in the literature to date. To the best of our knowledge, we are unaware of any work in which link quality prediction algorithms for MANETs are specifically designed for and evaluated in real-world urban environments. Our results emphasize the need for further research in this area, especially with regard to adaptive prediction models and techniques with which to deal with fast signal fading.

\section{Assessing Influencing Factors On Link Quality}

The poor performance of prediction algorithms in urban environments motivated us to examine systematically the factors that influence link quality and its prediction in 802.11 MANETs. The results are summarised in Table 2.

Table 2. Influence of various factors on link quality

\begin{tabular}{|l|l|l|l|l|l|l|}
\hline Factor & $\begin{array}{l}\text { Model of } \\
\text { wireless card }\end{array}$ & $\begin{array}{l}\text { Type of } \\
\text { ground }\end{array}$ & $\begin{array}{l}\text { Height } \\
\text { of nodes }\end{array}$ & $\begin{array}{l}\text { Orientation } \\
\text { without } \\
\text { shadowing body }\end{array}$ & $\begin{array}{l}\text { Orientation } \\
\text { with shadowing } \\
\text { body }\end{array}$ & $\begin{array}{l}\text { Shadowing } \\
\text { by person }\end{array}$ \\
\hline Influence & High & Low & High & Low & High & High \\
\hline \hline Factor & $\begin{array}{l}\text { Shadowing } \\
\text { by car }\end{array}$ & $\begin{array}{l}\text { Small scale } \\
\text { movements }\end{array}$ & $\begin{array}{l}\text { Large scale } \\
\text { movements at } \\
\text { different speeds }\end{array}$ & $\begin{array}{l}\text { Communication } \\
\text { load without } \\
\text { collisions }\end{array}$ & $\begin{array}{l}\text { Message } \\
\text { length }\end{array}$ & $\begin{array}{l}\text { Payload } \\
\text { pattern }\end{array}$ \\
\hline Influence & High & None & None & None & None & None \\
\hline
\end{tabular}

\subsection{Experimental Setup}

The manifold signal propagation phenomena observed in urban environments make the separation of potential and actual 'influencing factors' cumbersome in these environments. Hence we chose a beach on a deserted island as our 'benign' obstacle-free environment to conduct our experiments. We used the same hardware as for the urban environment study. Accurate markings and 
GPS receivers determined the distances between the two nodes that were always facing each other.

Three types of experiments were designed to assess potential and actual influencing factors on link quality:

Type I One node was motionless while the other node moved away at constant walking speed until returning after $300 \mathrm{~m}$. This type of experiment exhibits path loss and is for example suitable for comparing the theoretical Two-Ray Ground Model with actual measurements.

Type II Both nodes were placed at a fixed distance. One node was motionless while the other node oscillated between being in motion (e.g., node was rotated, shaken, etc.) and motionless. The comparison of the signal strength between the two different states reveals the influence of a factor.

Type III Both nodes were placed at a fixed distance and were motionless. An obstacle was moved in the line of sight between the nodes, paused and moved out. The difference in signal strength of the two states measures the influence.

All experiments were conducted three times and the mean was used for evaluation where possible. As described in Sect. 3.1] both nodes transmitted messages periodically. All diagrams display the signal strength that was measured at one node along the ordinate. If a packet was lost no value for the signal strength is given.

\subsection{Results}

Most simulations for MANETs (see 1314 for examples) are based on the TwoRay Ground Model with a maximum node transmission radius of $250 \mathrm{~m}$ for a transmission rate of 2 Mbit at maximum transmission power. Nevertheless, a recent experimental outdoor study from [28] claims that $250 \mathrm{~m}$ transmission range is far too optimistic and that the actual range would be 90 to 100 meters in an open environment. However, our measurements (Fig. 1 Type I experiment) confirm the widely accepted view of the literature that the transmission range is indeed around $250 \mathrm{~m}$. Our measurements confirm further that the Two-Ray Ground Model is a good approximation for open environments with a measured average model error of $1.49 \mathrm{dBm}$ (standard deviation $1.53 \mathrm{dBm}$ ).

These contradictory results may be attributed to the model of wireless card. In Fig. 2 (Type I experiment) we compare signal strength measurements of a Lucent Orinoco card with a card from a different brand. Both comply with the same 802.11 specification and have the same transmission power but show different behaviour. The observed performance discrepancy of the cards may be caused by different chip sets, the design of radio frequency components, different antennas and the applied signal processing of the cards' onboard processors. Unfortunately, no manufacturer provided us with the necessary information to enable an in-depth analysis. However, it should be noted that our comparison of 


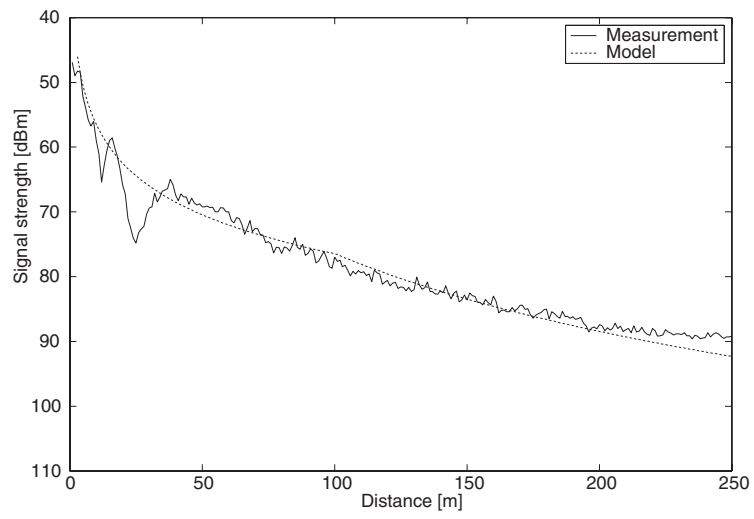

Fig. 1. Theoretical Two-Ray Ground Model and real-world measurements

simulation and real-world performance for different prediction algorithms in Sect. 3.3 is fair, even without detailed knowledge about the applied post-processing algorithms and hardware designs. As we have shown above, the measurements obtained using Lucent Orinoco cards correspond in an open environment very well with those given by the theoretical Two-Ray Ground Model on which the simulations are based on.

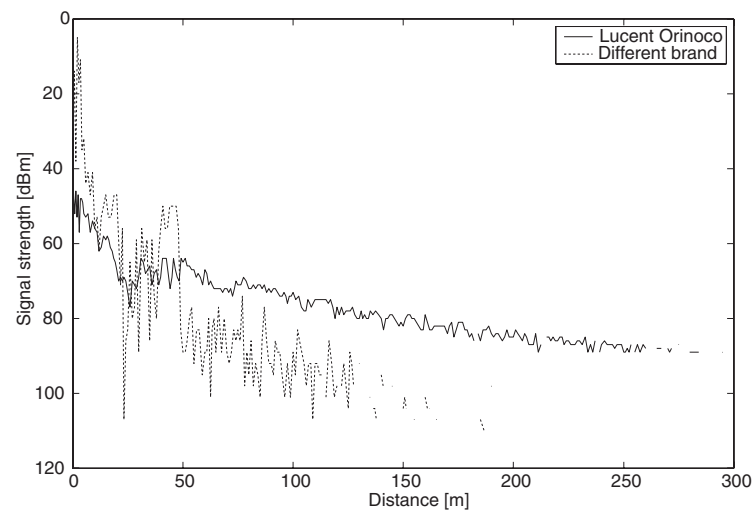

Fig. 2. Different card brands may show different behaviour

In all our trials we noted the persistent occurrence of a single but substantial 'dip' in the signal strength over approximately $10 \mathrm{~m}$ at distances ranging from $30 \mathrm{~m}$ to $50 \mathrm{~m}$ from the source (see for example Fig. 11). This is most likely due to coupling between the ground and the source (antenna) 29].

The influence of node height was assessed by placing two nodes $50 \mathrm{~m}$ apart while one node was stationary at navel height and the other changed its height as 
shown in Fig. B] (Type II experiment). The signal strength dropped significantly when one node was placed close to the ground and no relevant difference was observable between navel and head height. Since we assume that node heights change infrequently in ubiquitous computing scenarios, we rate the importance for link quality predictions in these applications to be low. However, link quality fluctuations caused by varying node heights may pose a problem for military or disaster recovery applications due to their mobility patterns.

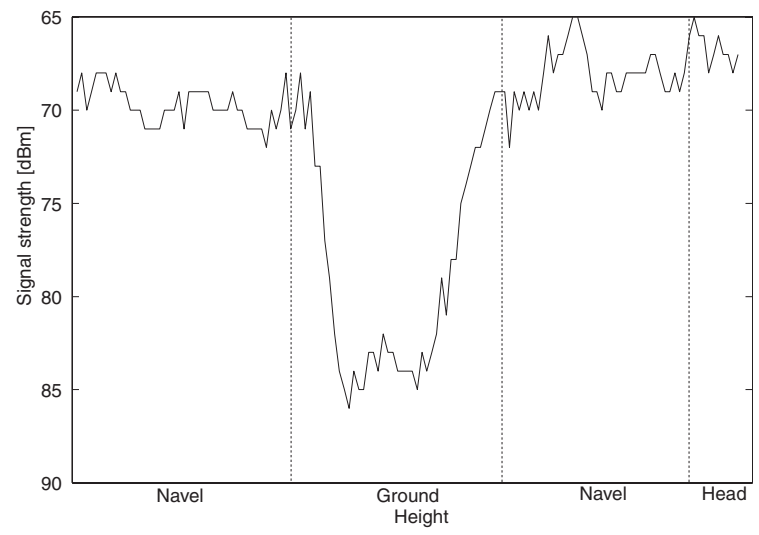

Fig. 3. Influence of node height on link quality

In personal wireless networks, the orientations of nodes change with the movements of the person (e.g. by turning) that holds the node. If standard hardware has a truly omni-directional antenna, this does not seem to pose a problem. However, we identified that the person's body can shadow the link severely based on its orientation and the node positions. This effect is illustrated at $150 \mathrm{~m}$ distance in Fig. 4 (Type II experiment). At around 180 degrees the body's shadowing caused a significant drop in the signal strength (a node rotation without a shadowing body caused no relevant changes in the signal strength). This leads to a link failure although the node could move $100 \mathrm{~m}$ further away at a different orientation without loosing the connection. Since the orientation changes frequently and suddenly in personal wireless networks, inevitable prediction errors in even the most benign open-space environment are the consequence.

People and cars are common in urban environments. We evaluate their influence on link quality by moving a person or car into the line of sight between two $100 \mathrm{~m}$ apart nodes (Type III experiment). Figure 5 and Fig. 6 present the influence of shadowing at different distances from the sender for a person and a car. At $20 \mathrm{~m}$ distance a person exhibits virtually no influence while a significant drop of link quality can be noticed $1 \mathrm{~m}$ away. A person directly in front of a node, which is common in crowds, leads even to a link failure at $100 \mathrm{~m}$ node distance. Similar but stronger link quality degradations can be observed for cars, where the connection was already lost at a shadowing distance of $1 \mathrm{~m}$. 


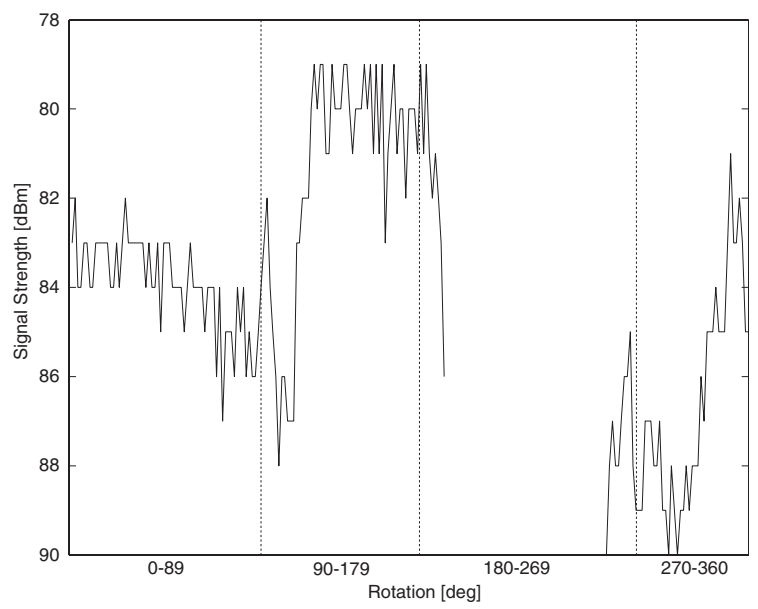

Fig. 4. Person shadowing a node with the own body while changing the orientation

We noticed that shadowing due to people and cars may degrade link quality by up to $30 \mathrm{dBm}$. Since we assume that the number and location of these objects are unpredictable, shadowing will always cause a significant amount of inevitable prediction errors. It should be noted that this result is not trivial since shadowing is a very important factor in 802.11 MANETs with more adverse effects on link quality than in traditional wireless networks (e.g. cellular phone networks). These traditional networks rely on an infrastructure with single-hop communication to base stations with antennas high over the ground (e.g. on building tops). Therefore the number of people in the line of sight is generally less than in 802.11 MANETs, where the antennas are usually located at either belt or car height. The infrastructure of cellular networks also provides a well defined coverage and well known reference points while in 802.11 MANETs no such reference points exists due to the mobility of all nodes. Moreover, cell phones can dynamically increase the transmission power to a much higher level for compensation of shadowing than 802.11 devices. 802.11 devices are restricted in their maximum transmission power to $15 \mathrm{dBm}$ due to the license free band. Furthermore the diffracting ability of 802.11 signals is lower due to the much higher operating frequency.

Other factors that influence signal strength were also examined but found to be insignificant. These are also listed in Table 2

\section{Conclusions}

We have observed that popular link quality prediction algorithms for 802.11 MANETs achieve only a much lower accuracy in real-world urban environments than in simulations. The best performing algorithm failed to predict 18 to 54 percent of total packet loss while 12 to 43 percent of packets were erroneously 


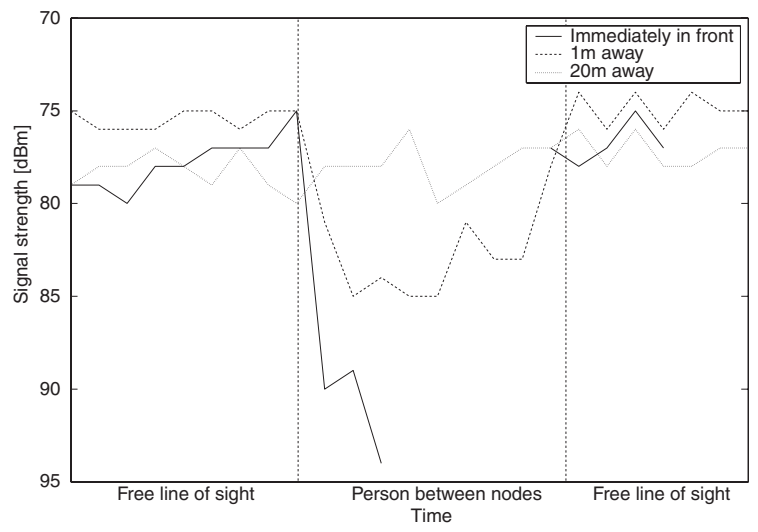

Fig. 5. Person shadowing a link at different distances

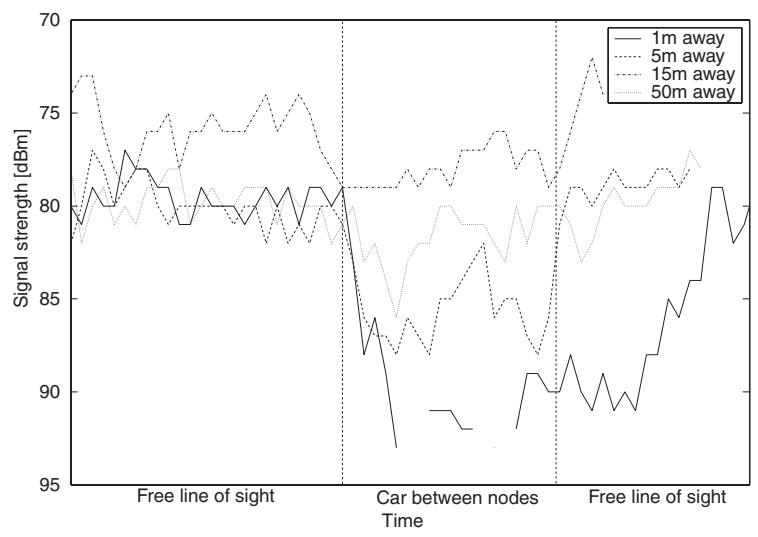

Fig. 6. Car shadowing a link at different distances

predicted to be lost. This contrasts sharply with near-perfect accuracy in simulations. The magnitude of this discrepancy has apparently not been appreciated in the literature to date.

We have observed that link quality prediction is especially difficult due to shadowing effects caused by user orientation, people and cars. The influence of such shadowing on link quality is much greater than in traditional cellular phone networks since in 802.11 MANETs there is no supporting infrastructure, transmission power is more limited and there is greater signal attenuation and poorer diffracting ability due to the higher operating frequencies used.

We identified, for low speed mobility scenarios, that algorithms based on the current received signal strength perform better than their trend-based counterparts. Current techniques to deal with fast signal fading were shown to be insufficient. Further research in this area is necessary. Moreover, we have determined that algorithm parameters are very specific to the actual operating 
environment. The accuracy of future prediction algorithms could be greatly improved by dynamically obtaining these parameters for the current operating environment.

Acknowledgments. The work described in this paper was partly supported by the Future and Emerging Technologies programme of the Commission of the European Union under research contract IST-2000-26031 (CORTEX - COoperating Real-Time sentient objects: architectures and EXperimental evaluation).

\section{References}

1. Scheideler, C.: Models and techniques for communication in dynamic networks. In: 19th Symposium on Theoretical Aspects of Computer Science, Antibes Juanles-Pins, France (2002) 27-49

2. Takai, M., Martin, J., Bagrodia, R.: Effects of wireless physical layer modeling in mobile ad hoc networks. In: International Conference on Mobile Computing and Networking, Proceedings of the 2001 ACM International Symposium on Mobile ad hoc networking and computing, Long Beach, CA, USA, ACM Press (2001) 87-94

3. Cavin, D., Sasson, Y., Schiper, A.: On the accuracy of MANET simulators. In: Proceedings of the Workshop on Principles of Mobile Computing (POMC'02), ACM Press (2002) 38-43

4. Heidemann, J., Bulusu, N., Elson, J., Intanagonwiwat, C., Lan, K., Xu, Y., Ye, W., Estrin, D., Govindan, R.: Effects of detail in wireless network simulations. In: SCS Multiconference on Distributed Simulation, Phoenix, Arizona, USA (2001) 3-11

5. Neskovic, A., Neskovic, N., Paunovic, G.: Modern approaches in modeling of mobile radio systems propagation environment. IEEE Communications Surveys 3 (2000) 2-12

6. Dube, R., Rais, C.D., Wang, K.Y., Tripathi, S.K.: Signal stability-based adaptive routing (SSA) for ad hoc mobile networks. IEEE Personal Communications 4 (1997) $36-45$

7. Paul, K., Bandyopadhyay, S., Mukherjee, A., Saha, D.: Communication-aware mobile hosts in ad-hoc wireless network. In: International Conferenceon Personal Wireless Communications (ICPWC'99), Jaipur, India (1999) 83-87

8. Punnoose, R.J., Nikitin, P.V., Broch, J., Stancil, D.D.: Optimizing wireless network protocols using real-time predictive propagation modeling. In: IEEE Radio and Wireless Conference (RAWCON), Denver, CO, USA (1999)

9. Lee, S.J., Su, W., Gerla, M.: Ad hoc wireless multicast with mobility prediction. In: IEEE ICCCN'99, Boston, MA, USA (1999) 4-9

10. Agarwal, S., Ahuja, A., Singh, J.P., Shorey, R.: Route-lifetime assessment based routing (RABR) protocol for mobile ad-hoc networks. In: IEEE International Conference on Communications. Volume 3., New Orleans (2000) 1697-1701

11. Jiang, S., Hez, D., Raoz, J.: A prediction-based link availability estimation for mobile ad hoc networks. In: IEEE INFOCOM, The Conference on Computer Communications, Twentieth Annual Joint Conference of the IEEE Computer and Communications Societies. Volume 3., Anchorage, Alaska, USA, IEEE (2001) 1745-1752

12. Su, W., Lee, S.J., Gerla, M.: Mobility prediction and routing in ad hoc wireless networks. International Journal of Network Management 11 (2001) 3-30 
13. Goff, T., Abu-Ghazaleh, N.B., Phatak, D.S., Kahvecioglu, R.: Preemptive routing in ad hoc networks. In: MOBICOM 2001, Proceedings of the seventh annual international conference on Mobile computing and networking, Rome, Italy, ACM Press (2001) 43-52

14. Qin, L., Kunz, T.: Pro-active route maintenance in DSR. ACM SIGMOBILE Mobile Computing and Communications Review 6 (2002) 79-89

15. McDonald, A.B., Znati, T.: A mobility-based framework for adaptive clustering in wireless ad-hoc networks. IEEE Journal on Selected Areas in Communication 17 (1999)

16. Roman, G.C., Huang, Q., Hazemi, A.: Consistent group membership in ad hoc networks. In: IEEE 23rd International Conference in Software Engineering (ISCE), Toronto, Canada (2001) 381-388

17. Gerharz, M., Waal, C.d., Frank, M., Martini, P.: Link stability in mobile wireless ad hoc networks. In: 27th Annual IEEE Conference on Local Computer Networks (LCN'02), Tampa, Florida (2002) 30-42

18. McDonald, A.B., Znati, T.: A path availability model for wireless ad-hoc networks. In: IEEE Wireless Communications and Networking Conference 1999 (WCNC '99), NewOrleans, LA, USA (1999)

19. Rappaport, T.S.: Wireless communications - principles and practice. 2nd edn. Prentice Hall Communications Engineering and Emerging Technologies Series. Prentice Hall (2002)

20. He, D., Shengming, J., Jianqiang, R.: A link availablity prediction model for wireless ad hoc networks. In: Proceedings of the International Workshop on Wireless Networks and Mobile Computing, Taipei, Taiwan (2000)

21. McCanne, S., Floyd., S.: UCB/LBNL/VINT network simulator - ns (version 2). http://www.isi.edu/nsnam/ns (1999)

22. Bajaj, L., Takai, M., Ahuja, R., Tang, K., Bagrodia, R., Gerla., M.: Glomosim: A scalable network simulation environment. Technical report, UCLA Computer Science Department (1999)

23. Johnson, D., Maltz, D.: Dynamic source routing in ad hoc wireless networks. In Imelinsky, Korth, H., eds.: Mobile computing. Kluwer Academic Publishers (1996) 153-181

24. Tripathi, N.D., Reed, J.H., Van Landingham, H.F.: Handoff in cellular systems. IEEE Personal Communications 5 (1998) 26-37

25. Veeravalli, V.V., Kelly, O.E.: A locally optimal handoff algorithm for cellular communications. IEEE Transactions on Vehicular Technology 46 (1997) 603-609

26. Gibson, D.: Orinoco_cs. http://ozlabs.org/people/dgibson/dldwd/ (2003)

27. Storn, R., Price, K.: Differential evolution a simple and efficient heuristic for global optimisation over continuous spaces. Journal of Global Optimization 11 (1997) 341-359

28. Anastasi, G., Borgia, E., Conti, M., Gregori, E.: IEEE 802.11 ad hoc networks: performance measurements. In: Workshop on Mobile and Wireless Networks (MWN 2003) in conjunction with ICDCS 2003. (2003)

29. Janaswamy, R.: A fredholm integral equation approach to propagation over irregular terrain. In: Antennas and Propagation Society International Symposium. Volume 2. (1992) 765-768 